Research Article

\title{
In silico characterization of microRNAs-like sequences in the genome of Paracoccidioides brasiliensis
}

\author{
Juliana S. de Curcio ${ }^{1,2}$ (iD), Mariana P. Batista ${ }^{1}$ (D), Juliano D. Paccez ${ }^{1}$ (D), Evandro Novaes ${ }^{3}$ (iD) and Célia \\ Maria de Almeida Soares ${ }^{1}$ iD \\ ${ }^{1}$ Laboratório de Biologia Molecular, Instituto de Ciências Biológicas, Universidade Federal de Goiás, \\ Campus II Samambaia, Goiânia, GO, Brazil. \\ ${ }^{2}$ Programa de Pós-Graduação em Patologia Molecular, Faculdade de Medicina, Universidade de Brasília, \\ Brasília, DF, Brazil. \\ ${ }^{3}$ Departamento de Biologia, Universidade Federal de Lavras, Minas Gerais, Brazil.
}

\begin{abstract}
Eukaryotic cells have different mechanisms of post-transcriptional regulation. Among these mechanisms, microRNAs promote regulation of targets by cleavage or degradation of the mRNA. Fungi of the Paracoccidioides complex are the etiological agents of the main systemic mycosis of Latin America. These fungi present a plasticity to adapt and survive in different conditions, and the presence of microRNAs-like molecules could be part of the mechanisms that provide such plasticity. MicroRNAs produced by the host influence the progression of this mycosis in the lungs besides regulating targets involved in apoptosis in macrophage, activation of $\mathrm{T}$ and $\mathrm{B}$ cells and the production of cytokines. Therefore, this work analyzed the presence of regions in the genome of this fungus with a potential to encode microRNAs-like molecules. Here we show by analysis of sequence similarity the presence of 18 regions, putatively coding for microRNAs-like molecules in the Paracoccidioides brasiliensis genome. We also described the conservation of dicer and argonaut proteins and the cognate transcripts induced in the yeast parasitic phase. This work represents a starting point for the analysis of the presence of those molecules in the morphological stages of the fungus and their role in fungal development.
\end{abstract}

Keywords: Fungi, mycoses, Dicer, Argonaute, microRNAs.

Received: January 15, 2018; Accepted: May 22, 2018.

\section{Introduction}

MicroRNAs were originally identified in Caenorhabditis elegans (Lee et al., 1993), and since then have been described in animals, plants and algae (Zhao et al., 2007; Grimson et al., 2008). In fungi, the first identified miRNAs-like molecules were described in Neurospora crassa (Lee et al., 2010). Subsequently, several studies demonstrated their presence in different species of fungi, such as Metarhizium anisopliae, Sclerotinia sclerotiorum, Penicillium marneffei, Trichoderma reesei, Fusarium oxysporum, Aspergillus flavus and Penicillium chrysogenum (Zhou et al., 2012a,b Kang et al., 2013; Lau et al., 2013; Chen et al., 2014; Bai et al., 2015; Dahlmann and Kück, 2015). In fungi such as $N$. crassa, different pathways are involved in miRNAs-like production. The production of milR-1 is dependent on the presence of of Dicer and the argonaut protein qde- $2 p$, while the biogenesis of

Send correspondence to Célia Maria de Almeida Soares. Laboratório de Biologia Molecular, Instituto de Ciências Biológicas, Universidade Federal de Goiás, Campus II Samambaia, Setor Itatiaia, 74001970 - Goiânia, GO, Brazil. E-mail: cmasoares@ gmail.com
milR-2 is independent of Dicer, but catalytic activity of argonaut qde- $2 p$ is required for the production of the premilRNA and the mature milR-2 (Lee et al., 2010).

MicroRNAs-like molecules influence the process of host-pathogen interaction (Zhou et al., 2012a; Lau et al., 2013). During mycosis development, several host microRNAs are regulated in response to the presence of these pathogens. Some of the regulated processes are macrophage polarization, chemokine expression, granulocyte production, inhibition of the Th1 immune response, and negative regulation of monocyte differentiation (reviewed in Croston et al., 2018). Analysis of the expression pattern of microRNAs produced by dendritic cells (DCs) after contact with A. fumigatus and Candida albicans revealed a specific response of microRNAs to the infection caused by these fungi. Some of the microRNAs induced after contact with these pathogens were miR-212 and miR132. These microRNAs are able to promote fine-tuning of mRNA expression involved in the immune response as, for example, $B T N 3 A 2$ a gene associated with the stimulation of the adaptive immune response in DCs, as well as of $F K B P 1 B$, which is associated with $\mathrm{T}$ cell proliferation in 
mice, and of KLF4 that is involved in the response of DCs to fungal infections (Dix et al., 2017). During the process of germination of A. fumigatus conidia in lungs, different levels of microRNA and mRNA expression, were observed in the host. Repressed microRNAs in lungs infected with conidia included miR-29a-3p, miR-30c-5p, and targets of these microRNAs were genes involved in the inflammatory response, such as $C l e c 7, S M A D 2 / 3$ and $T G F-\beta$. The gene Clec7a elicits an inflammatory response due to the recognition of $\beta$-glucan on the hypha cell wall. The TGF- $\beta$ signaling pathway includes the transcription factors from SMAD family, and microRNAs that regulate these transcripts were seen repressed in lung tissue. This demonstrated that conidia of A. fumigatus repress host microRNAs, and doing so, allow the expression of host mRNAs involved in the inflammatory response (Croston et al., 2016).

Paracoccidioidomycosis (PCM) is an endemic systemic mycosis caused by fungi of the genus Paracoccidioides. This genus comprises five species, $P$. brasiliensis, $P$. lutzii, $P$. americana, $P$. restrepiensis and $P$. venezuelensis (Restrepo, 1985, Turissini et al., 2017). It is estimated that approximately $80 \%$ of the patients who acquire this disease are Brazilians, the remainder of the cases occur mainly in Colombia, Venezuela, Argentina and Ecuador. The lethality rate of this disease is $3 \%$ to $5 \%$, and the number of cases in Brazil per year varies from 3360 to 5600 (Martinez, 2017). In systemic infections, the presence of a pathogen often induces a significant change in the expression profile of circulating microRNAs in the host. In this context, Singulani et al. (2017) analyzed the expression profiles of microRNAs produced by patients with PCM and compared these to those of healthy individuals. The data indicated the induction of expression of eight microRNAs among the individuals with PCM. Apoptosis of macrophages, activation of $\mathrm{T}$ and $\mathrm{B}$ cells, and fungal adhesion to host cells are processes that are putatively regulated by differentially expressed microRNAs, thus suggesting an influence of these molecules on the pathogen-host interaction process. Also, histopathological changes observed in the lungs of mice infected with $P$. brasiliensis at 28 and 56 days post-infection, correlated with the level of expression of the microRNAs produced by this organ. Within 28 days of infection, the lungs showed the presence of granulomas, with infiltrates of giant cells bordering the fungal cells, and the presence of phagocytic cells. At 56 days post-infection, the lungs presented a low number of yeast cells, characteristic of the resolution of the infection. Additionally, microRNAs induced at 56 days post-infection may act to reduce damage to lung tissues; for example the miRNA-26b-5p, which negatively regulates the expression of IL-6, could influence the amount of $\mathrm{T}$ cells at 56 days post- infection (Marioto et al., 2017).

Although miRNAs-like molecules have already been described in pathogenic microorganisms, as described above and microRNAs produced by the host are modulated in response to fungal infections, the presence of
microRNAs-like molecules in the genus Paracoccidioides is not described. In this work, we sought to identify proteins in the genomes of members of the Paracoccidioides genus that are involved in the post-transcriptional gene silencing pathway mediated by miRNAs. We evaluated the level of expression of transcripts encoding such proteins in the parasitic phase of $P$. brasiliensis. Using miRNA-like sequences identified in the fungi $N$, crassa, $C$. neoformans and $P$. marneffei, we performed an in silico identification of miRNAs-like sequences in the genome of $P$. brasiliensis. We identified dicer and argonaut proteins that are conserved in species of the Paracoccidioides complex, as well as sequences in the genome of $P$. brasiliensis that are predicted as microRNAs-like genes. To our knowledge, this is the first in-depth in silico analysis complemented with experimental results reporting the presence of microRNAlike coding regions in this important human pathogen.

\section{Material and Methods}

\section{In silico analysis of protein homologs in \\ Paracoccidioides spp. involved in the post-transcriptional gene silencing pathway}

The sequences of dicer and argonaute proteins present in the UNIPROT database were used for searching for homologous proteins in Paracoccidioides spp. We compared these sequences with those described for other fungal species: N. crassa, C. neoformans H99, Histoplasma capsulatum, Aspergillus nidulans and A. fumigates (Galagan et al., 2003; Nakayashiki et al., 2006; Janbon et al., 2010). Prediction of similarity and identity among homologous proteins was performed using the BLASTp tool implemented in the NCBI database. Sequence alignment and protein domain prediction were performed using CLUSTALX2 and the PFAM databases, respectively (Larkin et al., 2007; Finn et al., 2015).

\section{Phylogenetic analysis of RNA-induced silencing complex proteins in fungi}

The RNA-induced silencing complex proteins dicer and argonaute from Paracoccidioides spp., $N$. crassa, $C$. neoformans $\mathrm{H} 99, H$. capsulatum, A. nidulans, A. fumigatus were used for phylogenetic analysis. A phylogenetic tree was constructed by multiple sequence alignments using CLUSTALX2. The tree was generated by the neighborjoining method and visualized by TreeView software (Saitou and Nei 1987; Thompson et al., 1997). Branch robustness was estimated using 10,000 bootstrapped replicates. The accession numbers for each protein used in this comparison are described in Table S1.

\section{Culture and maintenance of $P$. brasiliensis and RNA extraction}

P. brasiliensis (ATCC 32069) yeast cells were cultivated in Sabouraud medium at $36^{\circ} \mathrm{C}$ (Sharma et al., 2010). After five days of growth in solid medium, yeast cells were 
inoculated in liquid medium and cultivated for $18 \mathrm{~h}$ at $36^{\circ} \mathrm{C}$ at $150 \mathrm{rpm}$. RNA from yeast cells cultured on this condition was extracted using the Trizol method (Silva-Bailão et al., 2014).

\section{Detection of transcripts from the RNA-induced silencing complex in $P$. brasiliensis}

The detection of transcripts involved in the posttranscriptional gene silencing pathway was performed by qRT- PCR. The primer sequences for the amplification of Dicer 1 (dcr 1), Dicer 2 (dcr 2), Argonaute 1 (ago-1), Argonaute 2 (ago-2) and Actin (act) are described in Table S2. In brief, total RNA extracted from $P$. brasiliensis was treated with DNase (RQ1 RNase-free DNase, Promega) and subjected to reverse transcription (SuperScript III First-Strand Synthesis SuperMix; Invitrogen, Life Technologies) following the manufacturer's recommendation. SYBR green PCR master mix (Applied Biosystems, Foster City, CA) was used in the qRT-PCR assays performed in a Step OnePlus system (Applied Biosystems. Normalization of the $\mathrm{Ct}$ values was done using the gene encoding an actin protein (GenBank XP_010761942). Standard curves were generated by 1:5 dilution of the cDNA, and the relative expression levels of the transcripts were calculated using the standard curve method for relative quantification (Bookout et al., 2006).

\section{In silico prediction of miRNAs-like molecules in the $P$. brasiliensis genome}

For in silico prediction of miRNAs-like molecules in the genome of $P$. brasiliensis, a literature search was performed to obtain sequences of mature miRNAs-like described in other fungi, including $F$. oxysporum, $P$. marneffei, $A$. flavus, $T$. reesei, $M$. anisopliae, $C$. neoformans, $N$. crassa, and in extracellular vesicles of $C$. neoformans, C. albicans, Saccharomyces cerevisiae, and P. brasiliensis (Lee et al., 2010; Jiang et al., 2012; Zhou et al., 2012a and b; Lau et al., 2013; Kang et al., 2013; Chen et al., 2014; Peres da Silva et al., 2015; Bai et al., 2015).

The sequences of such miRNA-like molecules were subjected to Blastn comparison against the $P$. brasiliensis $\mathrm{Pb} 18$ genome, using the task blastn-short option of the Blastn program (Camacho et al., 2009). Using a threshold E-value $<0.10$, alignments were obtained with at least 16 bp, and identities above $95 \%$. The results were converted into GFF files by means of a custom script written in Perl. The GFF files contain information about the position of genes, or of any other element in the reference genome. The InrsectBED tool from the BEDTools package (Quinlan and Hall 2010) was employed for identifying the position of the BLAST hits in relation to the annotation of the $P$. brasiliensis genome. Only the sequences of mature miRNAs-like that were not recorded in gene regions were considered for further analysis. After these steps, a Fasta file containing the sequences of the mature miRNAs-like molecules that aligned to the genome of $P b 18$ in non-gene regions was generated. The data in Fasta file format was considered $35 \mathrm{bp}$ or $50 \mathrm{bp}$ above or below the alignment region.

These sequences were analyzed for secondary structure in the RNAfold database, seeking to identify those that form hairpins similar to those of known pre-miRNAs. Potential miRNA sequences were manually revised using the following parameters: (A) the minimum size of the premiRNA could not be less than 45 nucleotides; (B) the pre-miRNA folded into a perfect stem-loop hairpin secondary structure; (C) the values of the minimum free energy (MFE) of folding should be at least $-7 \mathrm{kcal} / \mathrm{mol}$; (D) maximum mismatch of six between the miRNA/miRNA*duplex, where $(*)$ means the similar-sized fragment derived from the opposing arm of the pre-microRNA. These criteria were adopted to predict true microRNAs-like candidates (Dehury et al., 2013). As pre-miRNA size is variable and unknown for some cases in our study, we adjusted the size of the retrieved regions upstream and downstream (35-50 bp) of the alignment to search for a typical miRNA hairpin structure with RNAfold database (Lorenz et al., 2011, 2016). Manual checking of real pre-microRNAs has already been used in other bioinformatics studies (Dehury et al., 2013). All MFE values were expressed as negative $\mathrm{kcal} / \mathrm{mol}$ and adjusted MFE (AMFE) represented the MFE of 100 nucleotides, calculated by (MFE length of RNA sequence) $x 100$. The minimal folding free energy index (MFEI) was calculated by the equation: MFEI = $\mathrm{AMFE} /(\mathrm{G}+\mathrm{C}) \%$ (Zhang et al., 2006).

\section{Polyadenylation, reverse transcription and poly $(\mathrm{T})$ adaptor RT-PCR}

After the in silico prediction of microRNAs-like in the genome of $P$. brasiliensis, five potential candidates were chosen for validation experiments, based on the highest MFE values: $P b$-milR-11/Supercontig_2.3:1128222-1128358(-); Pb-milR-7/ Supercontig_2.6:955763_955879(+); $\mathrm{Pb}$-milR-6/Supercontig_2.5:587199-587317(+) and $\mathrm{Pb}$ milR-1/Supercontig_2.14:171583_171670(+). We also chose a candidate with the lowest MFE value $P b$-milR4/Supercontig_2.12:21200_21275(-). Validation experiments of microRNAs-like candidates employed the polyA tail method (Balcells et al., 2011). Initially, yeast cells and mycelium of $P b 18$ were cultured in Sabouraud medium (Sharma et al., 2010) for $18 \mathrm{~h}$, and the RNA of both conditions was extracted using TRIzol reagent (Sigma). Total extracted RNA was treated with DNase (RQ1 RNase-free DNase, Promega). The treated total RNA $(3 \mu \mathrm{g})$ was polyadenylated with ATP by poly(A) polymerase (Biolabs) using $4 \mu \mathrm{l}$ of $10 \mathrm{x}$ polyA buffer, $4 \mu \mathrm{l}$ of $10 \mathrm{mM} \mathrm{ATP}, 0.4 \mu \mathrm{l}$ of polyA polymerase corresponding to 2 units, and $3 \mu \mathrm{g}$ of RNA. Water was added to complete the reaction to a final volume of $20 \mu \mathrm{l}$. The polyadenylation reaction was performed at $37^{\circ} \mathrm{C}$ for $75 \mathrm{~min}$ and $65^{\circ} \mathrm{C}$ for $25 \mathrm{~min}$, according to the manufacturer's instructions. After this step, the polyadenylated RNAs were submitted to reverse transcription 
with SuperScrip II Reverse Transcriptase (Invitrogen). cDNA synthesis was done using $0.8 \mu \mathrm{l}$ of $20 \mathrm{nM}$ poly(T) adapter, $4 \mu \mathrm{l}$ of $10 \mathrm{mM}$ dNTPS, $8 \mu \mathrm{l}$ of $5 \mathrm{x}$ buffer, $1.5 \mu \mathrm{l}$ of $25 \mathrm{mM} \mathrm{MgCl}_{2}, 0.5 \mu \mathrm{l}$ of RNAse inhibitors, $1.25 \mu \mathrm{l}$ of reverse transcriptase, and $5 \mu \mathrm{l}$ of polyadenylated RNA. The conditions for cDNA synthesis were: $42^{\circ} \mathrm{C}$ for $135 \mathrm{~min}$ and $85^{\circ} \mathrm{C}$ for $10 \mathrm{~min}$. Qualitative RT-PCR assays were performed at $94^{\circ} \mathrm{C}$ for $2 \mathrm{~min}$, followed by 40 cycles of $95^{\circ} \mathrm{C}$ for $15 \mathrm{~s}, 57^{\circ} \mathrm{C}$ or $57.6^{\circ} \mathrm{C}$ for $5 \mathrm{~s}$, and $72^{\circ} \mathrm{C}$ for $20 \mathrm{~s}$. and a final elongation step at $72^{\circ} \mathrm{C}$ for $5 \mathrm{~min}$. The amplification products were visualized on $3 \%(\mathrm{w} / \mathrm{v})$ agarose gels, and product size the potential microRNAs-like molecules was calculated by linear regression. The primers used for the amplification of microRNAs-like are listed in Table S2.

\section{Results}

In silico analysis indicates the presence of proteins involved in post-transcriptional gene silencing in Paracoccidioides spp.

A flowchart of the steps for in silico prediction of proteins involved in gene silencing by RNA interference and for potential miRNAs-like is shown in Figure S1. Our analysis allowed the identification of dicer and argonaute proteins in Paracoccidioides spp. (Table 1). P. brasiliensis (Pb18), P. americana (Pb03), and P. lutzii presented two dicer (Dcr-1p and Dcr-2p) and two argonaute (Ago-1 and Ago-2) proteins. C. neoformans Serotype D presented two paralogous genes for argonaute (agol and ago2) and dicer (dcr 1 and $d c r 2$ ), in contrast to the $C$. neoformans serotype A (H99), which contains a single gene for argonaute (agol) and two paralogous genes for dicers ( $d c r 1$ and $d c r 2)$ (Janbon et al., 2010). Studies with $N$. crassa demonstrated the presence of two genes that encode dicers (qde-2 and $d c r)$ and two that code for argonautes ( $\mathrm{Sms}_{\mathrm{s}} 2$ and $\mathrm{Sms}-3$ ) (Galagan et al., 2003). The $A$. nidulans genome has only one gene that codes for dicer (ANID_10380) and one that codes for argonaute (ANID_01519), while A. fumigatus has two homologs that encode for two dicer (AFUA_5G11790/AFUA_4G02930) and two argonaute proteins (AFUA_3G11010/AFUA_8G05280) (Nakayashiki et al., 2006; Janbon et al., 2010). Hence, the number of proteins involved in post transcriptional gene silencing in fungi of the Paracoccidioides complex is similar in number to other fungi. Furthermore, the analysis of identity indicates major similarity among proteins from Paracoccidioides spp., H. capsulatum, and A. fumigatus (Table 1).

The phylogenetic analysis of Ago-1p and Ago-2p, Dcl-1p and Dcl-2p showed that the sequences present in Paracoccidioides spp. are closely related to Blastomyces dermatittidis, $H$. capsulatum, $P$. chrysogenum, and Aspergillus spp. (Figure S2). With phylogenetic distances from 0.98 to 1 it is possible to infer that the proteins are orthologous, possibly having a similar function among dif- ferent fungi. Correspondingly, a study by Lau et al. (2013) showed that Dcl-2p of $P$. marneffeiis is more closely related to those of the thermal dimorphic pathogenic fungi $H$. capsulatum, $B$. dermatitidis, and $C$. immitis, than to $P$. chrysogenum and Aspergillus spp.

The post-transcriptional gene silencing machinery is highly conserved in Paracoccidioides spp. and other fungi

The prediction of protein domains performed using the PFAM database allowed the identification of conserved domains in dicers and argonauts (Figure 1). It is important to highlight that the main components involved in miRNA processing and gene silencing include RNA polymerase (Pol II), dicer, and the silencing complex induced by RNA (Bartel et al., 2004). Studies analyzing domains of the canonical dicer of $N$. crassa Dcr-2p demonstrated the presence of DEAD box, Helicase $\mathrm{C}$, ribonuclease 3 and dicer dimerization domains. Dicer 1 from $N$. crassa contains at its N-terminal region a RESIII domain instead of a DEAD box. The homologue from $C$. neoformans has only the ribonuclease 3 and dicer dimerization domains (Janbon et al., 2010), and the analysis of the Dcr-1p proteins from Pb18 and P. lutzii (Pb01) indicates similarity to the homologous protein found in $C$. neoformans, since it possesses only the dicer dimerization and the ribonuclease 3 domains (Figure1A). On the other hand, the analysis of the same homologue in $\mathrm{Pb03}$ demonstrated higher similarity to the dicer dimerization, ribonuclease 3 and RESIII domains found in the $N$. crassa homolog (Figure 1A). Therefore, we can conclude that the general domain arrangement of this protein is not fully conserved among members of the complex Paracoccidioides.

Furthermore, the analysis of protein domains from Paracoccidioides spp. demonstrated that Dcr- $2 p$ contains the Dicer dimerization, DEAD/DEAH box, Helicase C, and ribonuclease 3 domains similar to those present in other dimorphic and filamentous fungi, except for $H$. capsulatum (H143) (Figure 1B). Comparing the members of the Paracoccidioides complex, depicted in (Figure 1), it is apparent that Dcr-2p is conserved among species of Paracoccidioides.

Previous studies described the dicer dimerization domain as being similar to the binding domain of dsRNA (Dlakic, 2006), while the DEAD/DEAH-box helicase domain interacts with the pre-miRNA loop, facilitating the alignment of this molecule to the RNase III domain for precise pre-miRNA cleavage (Tsutsumi et al., 2011). Additionally, the Helicase $\mathrm{C}$ domain binds to the dsRNA loop region and checks the size. Furthermore, this domain also measures the distance between two adjacent nucleotides in the 3 'region of the mRNA molecule, with the loop region verifying the pre-miRNA sequence before cutting (Tsutsumi et al., 2011). Dicers proteins have a ribonuclease 3 domain responsible for the cleavage of pre-miRNA, generating a duplex of small RNAs with two adjacent nu- 


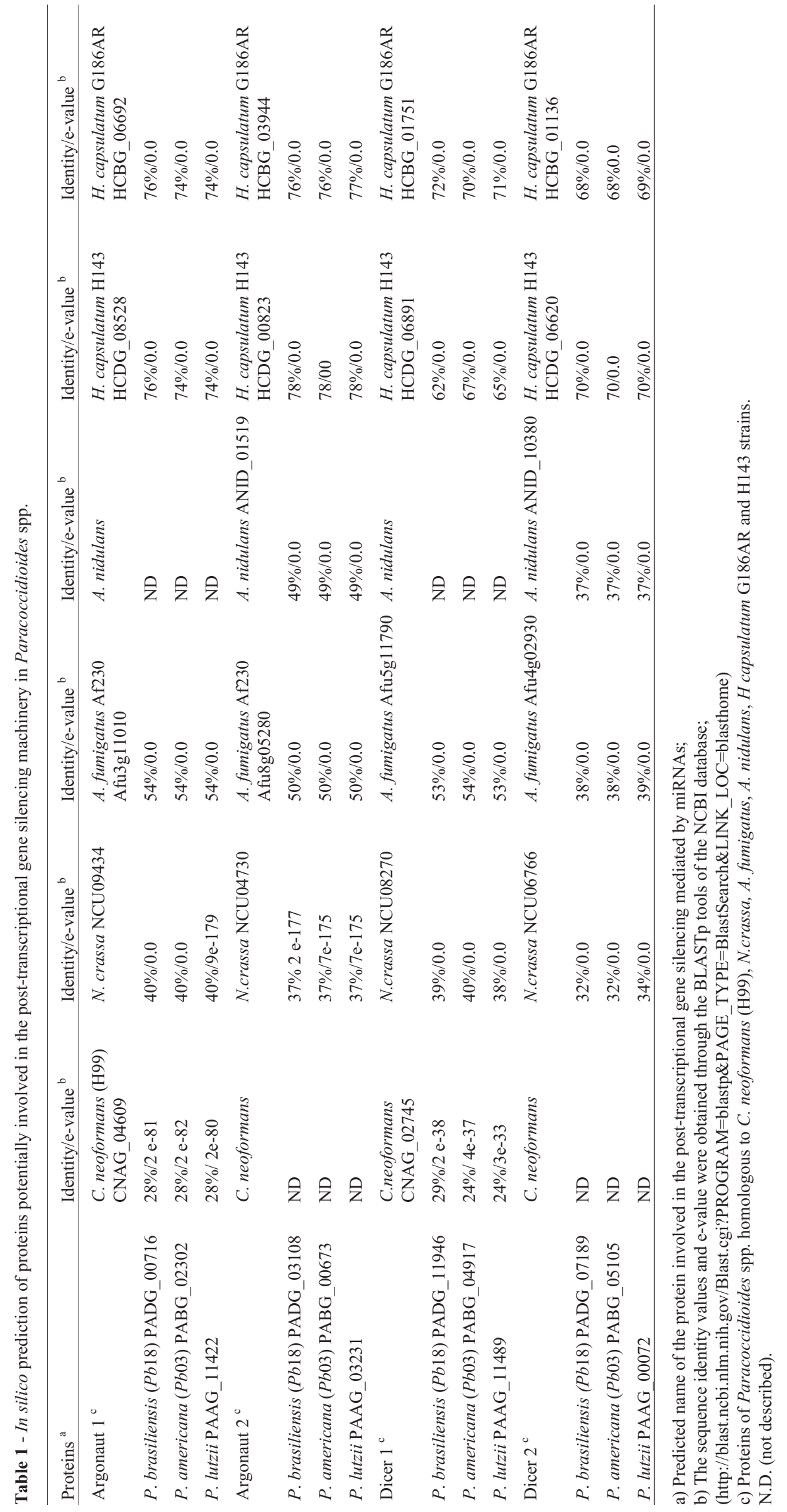



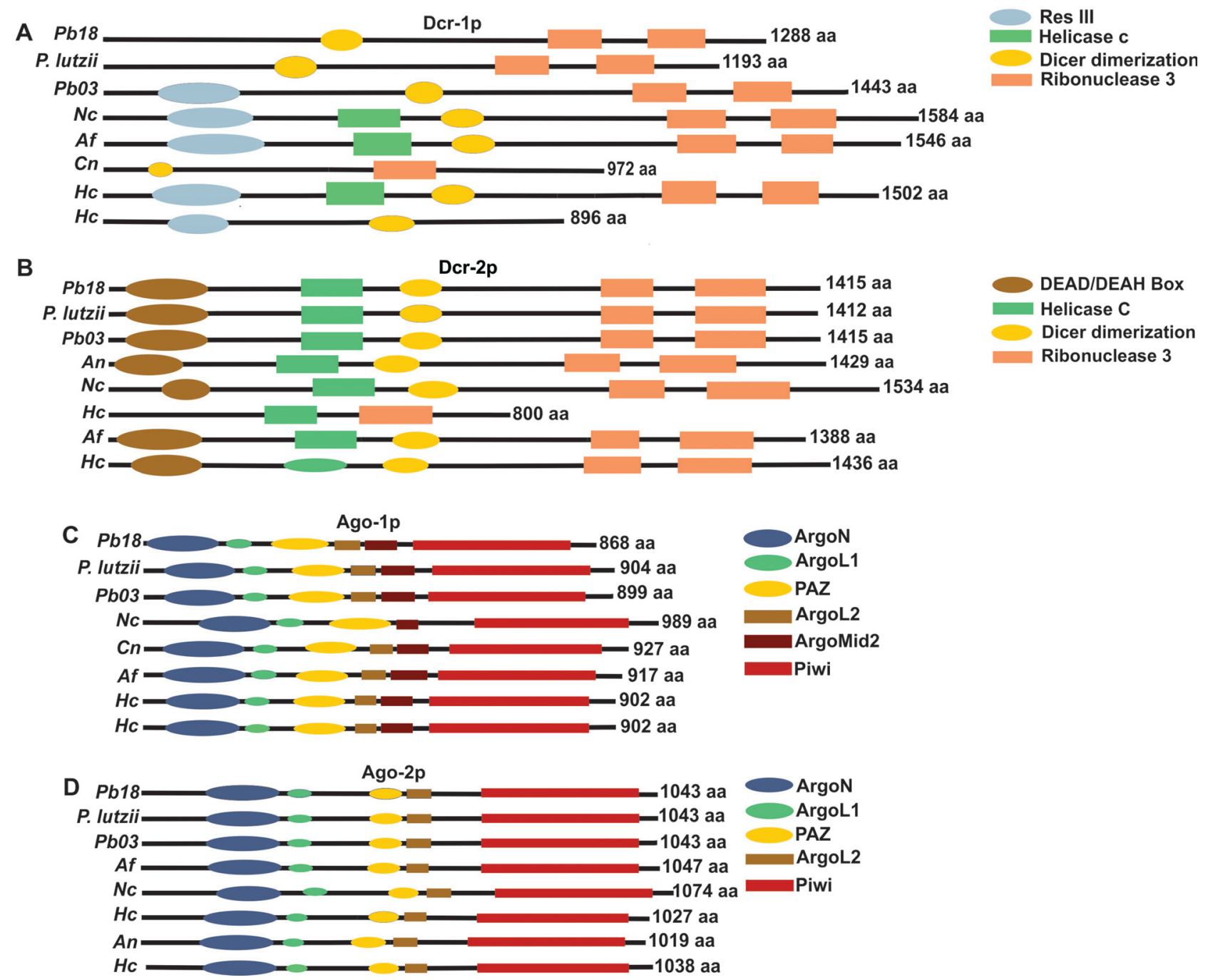

Figure 1 - Domains identified in proteins involved in the post-transcriptional gene silencing pathway. (AD) Domains present in dicers and argonauts proteins from P. brasiliensis $(\mathrm{Pb} 18)$, P. lutzii ( $\mathrm{Pb} 01)$ and P. americana $(\mathrm{Pb} 03)$. The accession numbers of the proteins are as follows. Dicer 1: (PADG_11946; PABG 04917; PAAG 11489; NCU08270; Afu5g11790; CNAG 02745; HCBG 01751; HCDG 06891), Dicer 2: (PADG 07189; PAAG 00072; PABG_05105; ANID_10380; NCU06766; HCDG_06620; Afu4g02930; HCBG_01136) Ago1: (PADG_00716; PAAG_11422; PABG_02302; NCU09434; CNAG 04609; Afu3g11010; HCBG 06692; HCDG 08528) Ago2: (PADG 03108; PAAG 03231; PABG 00673; Afu8g05280; NCU04730; HCBG_03944; ANID_01519; HCDG_00823).

cleotides in the 3'end (Zhang et al., 2004). In this regard, our data suggest that these proteins are able to process double-stranded RNA, since we identified conserved domains among dicers from Paracoccidioides spp.

Our data also indicate that Ago-1p from Paracoccidioides spp. contains PIWI, PAZ, ArgoL1, ArgoL2, ArgoMid2 and ArgoN domains (Figure1C), while Ago-2p contains PIWI, PAZ, ArgoL1, ArgoL2 and ArgoN domains (Figure1D). The analysis of argonaute proteins from $N$. crassa, P. chrysogenum, P. marneffei and C.neoformans indicated the presence of PAZ and PIWI domains that are conserved among different species of fungi and that play a role in RNA interference processes (Nakayashiki, 2005; Joshua-Tor, 2006; Janbon et al., 2010; Lau et al., 2013; Dahlmann and Kück, 2015). Interestingly, the PIWI domain present in argonaute shows a tertiary structure similar to the RNase $\mathrm{H}$ family and has been described as being involved in targeting mRNA cleavage, while the PAZ domain is responsible for the binding and transfer of small RNAs to the RISC complex (Lingel et al., 2003; Yan et al., 2003; Song et al., 2004).

After in silico prediction of proteins involved in gene silencing mediated by miRNAs-like molecules, the respective transcripts levels were quantified by qRT-PCR. We performed this study in $\mathrm{Pb} 18$, a highly investigated species in the Paracoccidioides complex. The transcripts were assessed in yeast cells cultivated at $18 \mathrm{~h}$ of growth. As shown in Figure 2, transcripts for dicers and argonauts are clearly detected after $18 \mathrm{~h}$ of growth. Detection of the transcripts encoding argonaute and dicer proteins reinforce our in silico data, depicting the active pattern of transcription of the cognate genes. 

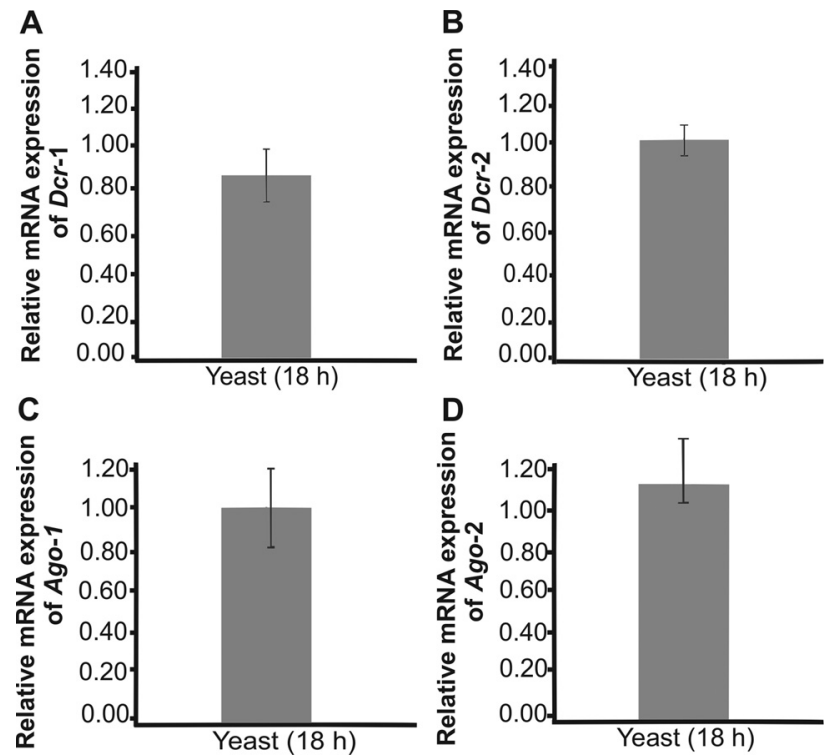

Figure 2 - Genes involved in the processing of microRNAs-like are induced in the parasitic phase of P. brasiliensis. Quantitative RT-PCR was performed with transcripts of Pb18 yeast cells cultivated in liquid Sabouraud medium for 18 hours. Expression values of genes involved in the processing of microRNAs ( $d c r-1, d c r-2$, ago-1, ago-2) were calculated using actin as the endogenous control. Data are expressed as mean ( standard deviation from triplicates.

\section{Identification of miRNAs-like sequences in the $P$.} brasiliensis Pb18 genome

In order to identify putative miRNAs-like in the genome of $P$. brasiliensis (Pb18) we analyzed the similarity between microRNA-like sequences characterized in fungi or present in vesicles secreted by these microorganism, with regions of the genome of $P$. brasiliensis Pb18. The miRNAs-like sequences were obtained from the literature. In the first step we analyzed the microRNAs-like characterized in other fungi, and from this analysis we identified 134 potential miRNAs-like sequences (Table S3). Remarkably, amongst these miRNA-like sequences, 20 sequences aligned in the genome of P.brasiliensis with identities higher than 95\% (Table S3). Moreover, the analyzed sequences demonstrated a high percentage of alignment with A. flavus $(7.5 \%)$ and $N$. crassa (5\%) genomes (Figure $\mathrm{S} 3$ ). Furthermore, we observed that seven sequences matched all the criteria (Dehury et al., 2013) to be considered mature miRNA-like molecules in the $P$. brasiliensis ( $\mathrm{Pb18}$ ) genome (Table 2). The analysis of the predicted secondary structure of these sequences corroborated the features of a pre-miRNA hairpin and free energy folding (Table 2 and Figure $3 \mathrm{~A}$ ). In fact, all the Fasta sequences were analyzed in relation to these two characteristics by the RNAfold program. In an analysis for miRNAs-like sequences described to be present in secreted vesicles (Peres da Silva et al., 2015) a total number of 1477 miRNA sequences was described in vesicles of other fungi species, but only 100 sequences were aligned in the genome of $P$. brasiliensis with identity higher than $95 \%$ (Table S4). It is important to note that in many cases, the same miRNAs-like sequence aligned in more than one region in the $P$. brasiliensis genome. From the total of 100 sequences that aligned in the genome of $P$. brasiliensis, only 11 presented all the criteria for potential miRNAs-like molecules (Dehury et al., 2013) (Table 2 and Figure 3B). The microRNAs-like predicted in silico for $P$. brasiliensis $P b 18$ that are already described in other fungi are listed in Figure 3A, whereas the microRNAs present in vesicles with similarity are shown in Figure 3B.

Our in silico prediction approach based on the comparison with miRNAs-like data described in other fungi or present in secreted vesicles allowed the characterization of 18 potential miRNAs-like sequences in the genome of $P$. brasiliensis ( $P b 18$ ), with identity values above $95 \%$, values of negative folding free energies between $-7.60 \mathrm{kcal} / \mathrm{mol}$ and $-33.30 \mathrm{kcal} / \mathrm{mol}$ (Tables 2 and 3), as well as evidence for hairpin formation. Previous reports have evaluated the MFEI and calculated the AMFE values (Zhang et al., 2006). In plants, MFEI values were found to vary between 0.81 and 0.96, and their AMFE values between 26 and 44 $\mathrm{kcal} / \mathrm{mol}$ (Mishra et al., 2015). We observed lower MFEI but similar AMFE values those described in plants (Table 3). We also evaluated other features of miRNA precursors, such as the amount of uracil and adenine in their sequences. In fact, previous reports demonstrated that miRNA precursors have a higher percentage of these bases in their sequences (Zhang et al., 2006). As demonstrated in (Table 3), the majority of the miRNA precursors described in $P$. brasiliensis have a high proportion of uracil and adenine in their sequence.

In general, the in silico analyses based on similarity of sequences allowed the identification of 18 potential microRNA-like coding regions. These exhibited microRNAs-like characteristics, such as hairpin structure, MFE values similar to those already described for microRNAs-like from other microorganisms, a large amount of uracil, demonstrating that these coding regions of microRNAs-like are conserved in the genome of $P$. brasiliensis.

\section{Experimental validation of predictions obtained by bioinformatics}

The validation of our data predicted by bioinformatics was performed by qualitative RT-PCR, for five potential microRNAs-like sequences (Figure 4). The selection of these microRNAs-like was based on the highest and lowest MFE values. The presence of three amplification products was detected representing, putatively, the pri-microRNA, pre-microRNA and mature microRNAlike molecules. This method has previously been used to detect microRNAs in different cell types (He et al., 2005; Chiba et al., 2012). Therefore, the presence of these amplification products confirms the data predicted by bioinformatics and suggests these potential microRNAs-like 


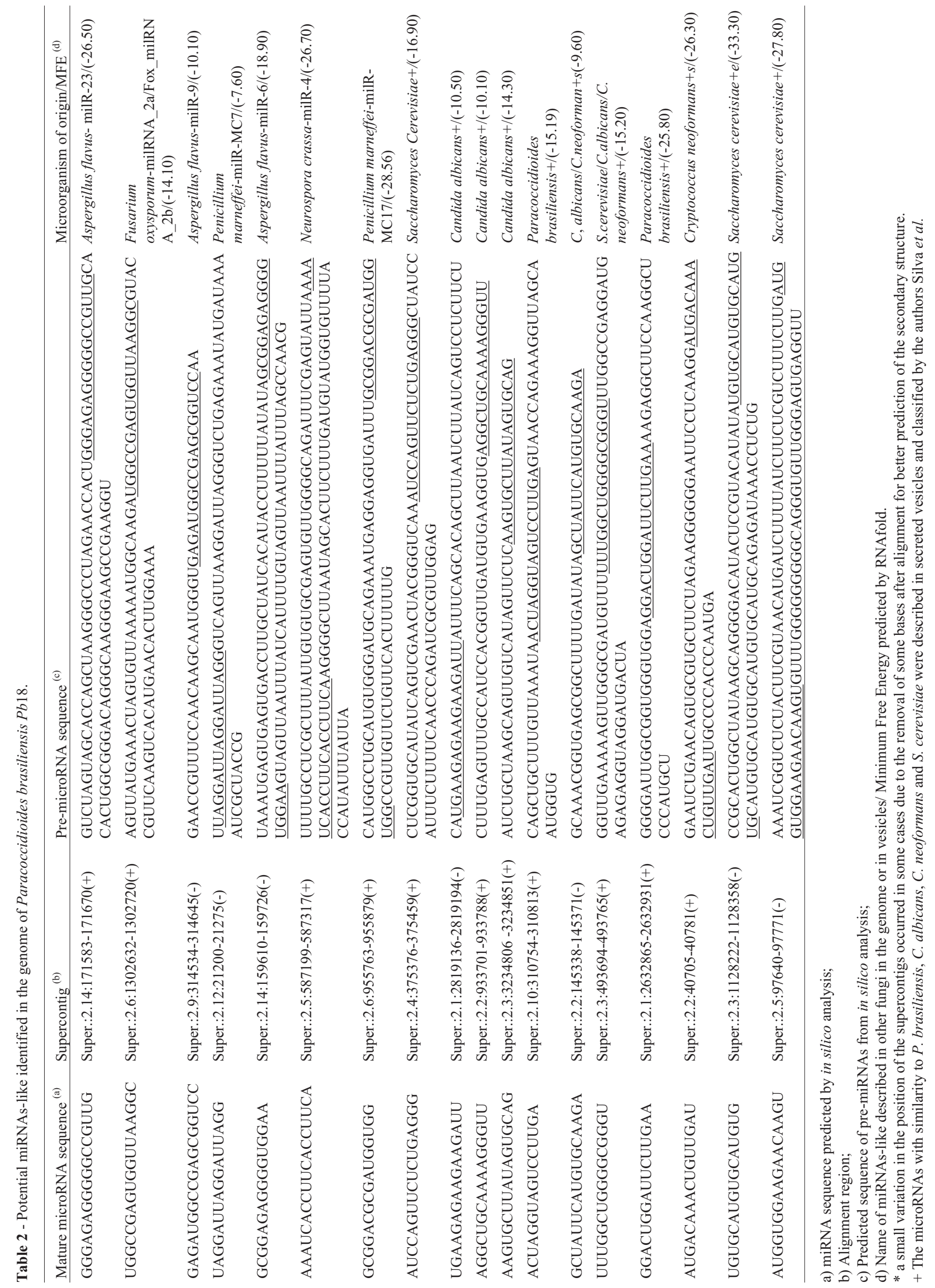




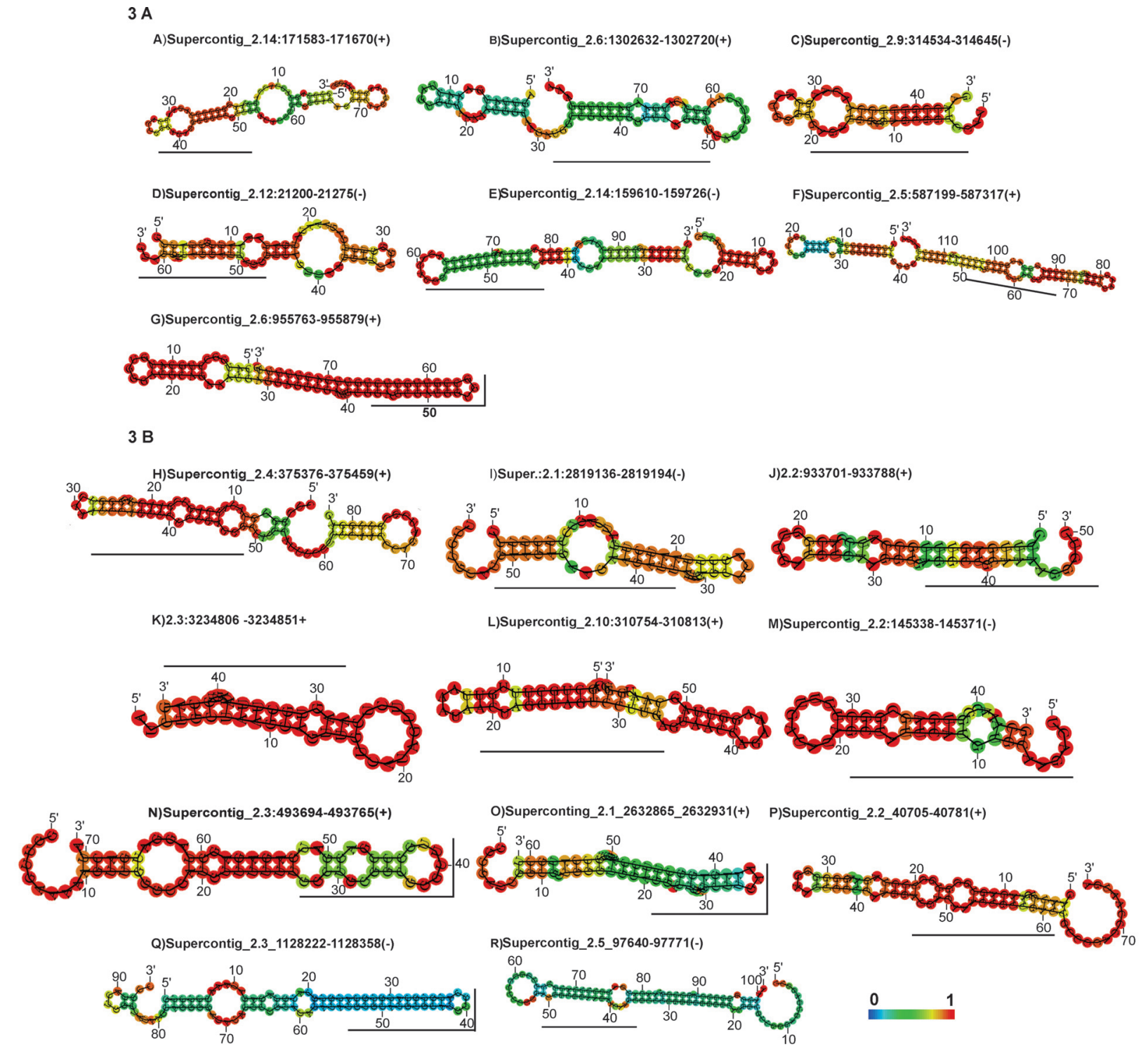

Figure 3 - Representation of the newly-identified potential pre-miRNAs in P. brasiliensis. The matured miRNA portion is highlighted in black bar. The structures were generated using the Rfold program. The actual size of the precursors may be slightly shorter or longer than shown in the figures. The structures are colored according to the base pairing probabilities. Red color denotes high probability, as represented in the color bar.

candidates as being conserved in the genome of this human pathogen.

\section{Discussion}

Proteins involved in the machinery of gene silencing by RNA interference (dicers and argonauts) are conserved in fungi of different phyla, including Ascomycota, Basidiomycota, and Zygomycota (Drinnenberg et al., 2009). Our analysis demonstrated that the number of proteins involved in the post-transcriptional gene silencing pathway of Paracoccidioides spp. are in line with data described in the literature for other fungi, such as $C$. neoformans, A. fumigatus, and $N$. crassa that also present two paralogous genes for dicer and argonaute in their genomes (Galagan et al., 2003; Nakayashiki et al., 2006;
Janbon et al., 2010). In this regard, the conservation of domains in proteins involved in RNA interference in Paracoccidioides spp. demonstrates that these organisms retain components involved in the processing of miRNAslike, as seen in other fungi (Lee et al., 2010; Jiang et al., 2012). In addition, proteins involved in the processing of microRNAs-like in Paracoccidioides spp. were seen to be phylogenetically related to dimorphic fungi, and, thus, are likely to perform similar functions (Lau et al., 2013). Furthermore, our gene expression data reinforce the suggested conservation of the post-transcriptional gene silencing machinery mediated by microRNAs and support the concept that these genes are active in the parasitic form of this pathogenic fungus. 
Table 3 - Characteristics of miRNAs-like molecules predicted in this study.

\begin{tabular}{|c|c|c|c|c|c|c|c|}
\hline Number of microRNA $^{a}$ & Supercontig $^{\mathrm{b}}$ & Length $^{\mathrm{c}}$ & $\mathrm{MFE}^{\mathrm{d}}$ & $\mathrm{AMFE}^{\mathrm{e}}$ & $\mathrm{MFEI}^{\mathrm{f}}$ & $(\mathrm{G}+\mathrm{C}) \%^{\mathrm{g}}$ & $(\mathrm{A}+\mathrm{U}) \%{ }^{\mathrm{h}}$ \\
\hline$P b$-milR-1* & Super.:2.14:171583-171670(+) & 17 & -26.50 & 30.45 & 0.48 & $63.2 \%$ & $36.80 \%$ \\
\hline$P b$-milR-2 & Super.:2.6:1302632-1302720(+) & 18 & -14.10 & 17.62 & 0.42 & $41.25 \%$ & $58.75 \%$ \\
\hline$P b$-milR-3 & Super.:2.9:314534-314645(-) & 18 & -10.10 & 22.44 & 0.40 & $55.6 \%$ & $44.44 \%$ \\
\hline$P b$-milR-4* & Super.:2.12:21200-21275(-) & 16 & -7.60 & 12.06 & 0.31 & $38.1 \%$ & $61.9 \%$ \\
\hline$P b$-milR-5 & Super.:2.14:159610-159726(-) & 16 & -18.90 & 18.9 & 0.55 & $34 \%$ & $66 \%$ \\
\hline$P b$-milR-7* & Super.:2.6:955763-955879(+) & 16 & -28.56 & 36.61 & 0.69 & $52.56 \%$ & $47.43 \%$ \\
\hline$P b$-milR-8 & Super.:2.4:375376-375459(+) & 17 & -16.90 & 20.36 & 0.41 & $49.50 \%$ & $50.60 \%$ \\
\hline$P b$-milR-9 & Super.:2.1:2819136-2819194(-) & 16 & -10.50 & 20.19 & 0.56 & $35.8 \%$ & $64.2 \%$ \\
\hline$P b$-milR-10 & Super.:2.2:933701-933788(+) & 16 & -10.10 & 19.80 & 0.4 & $49.00 \%$ & $51.00 \%$ \\
\hline$P b$-milR-11* & Super.:2.3:1128222-1128358(-) & 16 & -33.30 & 35.42 & 0.73 & $47.87 \%$ & $51.06 \%$ \\
\hline$P b$-milR-12 & Super.:2.10:310754-310813(+) & 16 & -15.19 & 25.74 & 0.63 & $40.67 \%$ & $59.32 \%$ \\
\hline$P b$-milR-13 & Super.:2.2:145338-145371(-) & 17 & -9.60 & 22.32 & 0.50 & $44.2 \%$ & $55.8 \%$ \\
\hline$P b$-milR-14 & Super.:2.3:493694-493765(+) & 16 & -15.20 & 21.4 & 0.41 & $52.11 \%$ & $47.88 \%$ \\
\hline$P b$-milR-15 & Super.:2.1:2632865-2632931(+) & 16 & -25.80 & 42.29 & 0.73 & $57.40 \%$ & $42.60 \%$ \\
\hline$P b$-milR-16 & Super.:2.2:40705-40781(+) & 16 & -26.30 & 34.60 & 0.71 & $48.60 \%$ & $51.40 \%$ \\
\hline$P b$-milR-17 & Super.:2.3:3234806-3234851(+) & 17 & -14.30 & 32.5 & 0.79 & $40.90 \%$ & $59.1 \%$ \\
\hline$P b$-milR-18 & Super.:2.5:97640-97771(-) & 17 & -27.80 & 27.52 & 0.58 & $47 \%$ & $54 \%$ \\
\hline
\end{tabular}

Name of predicted microRNA-like in the genome of $P$. brasiliensis ( $P b 18)$;

Region of alignment;

Size of mature microRNA-like;

Minimum free energy predicted by RNA fold;

AMFE: adjusted minimal free folding energy ( $\mathrm{kcal} / \mathrm{mol})$;

MFEI: minimal free folding energy index;

Percentage of nucleotides $(\mathrm{G} / \mathrm{C})$ present in the pre-microRNA sequence;

Percentage of nucleotides $(\mathrm{A} / \mathrm{U})$ present in the pre-microRNA sequence;

* microRNAs-like used for the validation experiments.
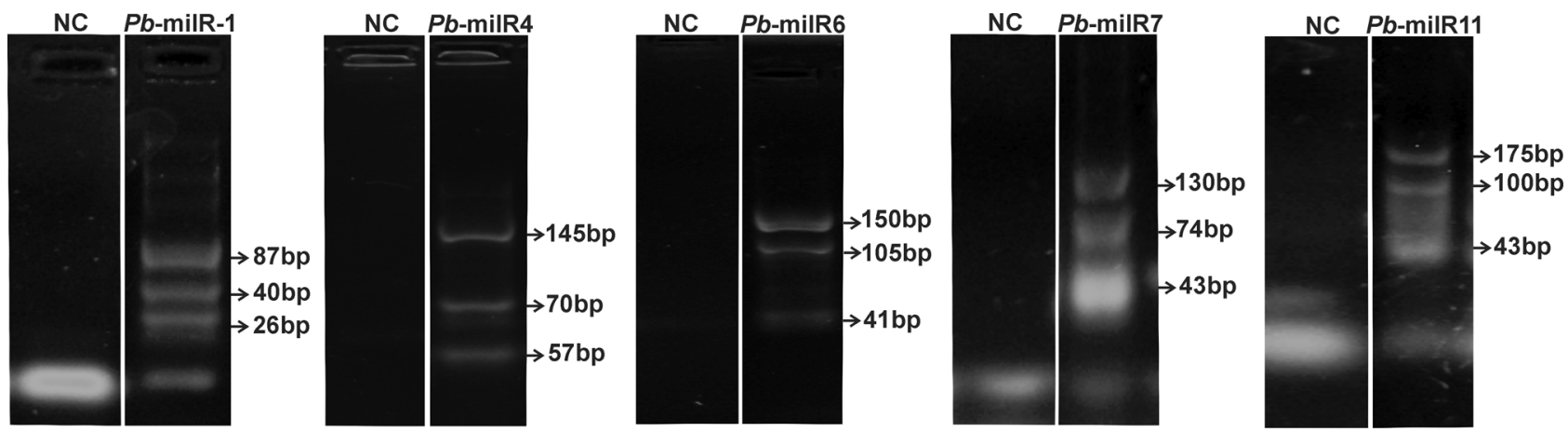

Figure 4 - Qualitative RT-PCR of microRNAs-like predicted by bioinformatics tools. Five potential microRNAs-like were selected on the basis of the highest and lowest MFE values and were used for the validation experiments. Each reaction sample was individually fractionated on a $3 \%$ agarose gel and the fragments sizes were predicted by linear regression. RNA was obtained from P. brasiliensis $\mathrm{Pb} 18$.

Different dicer and argonaut proteins are required to produce microRNAs-like molecules in fungi such as $N$. crassa (Lee et al., 2010). These proteins are specifically involved in the production of microRNAs-like molecules in mycelium and yeast cells. For example, the dicer 2 protein is specifically involved in the production of two microRNAs-like molecules exclusive of the mycelium phase of $P$. marneffei (Lau et al., 2013). In addition, microRNAs-like molecules can also be processed via the canonical pathway, involving both both dicer and argonaut proteins (Lee et al., 2010). Our data demonstrate the expression of dicers and argonauts in the yeast phase of $P$. brasiliensis, and in this same condition, the expression of five microRNAs-like was identified. Therefore, the expression of the transcripts encoding the proteins involved in microRNAs processing, possibly correlates with the identified small RNAs, since the transcripts encoding these proteins were also induced in the yeast phase. However, which proteins are specifically involved in the production of these microRNAs-like molecules still needs to be investigated. 
The in silico prediction of microRNAs-like molecules in P. brasiliensis demonstrated that this fungus retains regions in the genome that are responsible for the genesis of mature miRNAs. Mature sequences of miRNAs conserved in different kingdoms are already described in the literature, demonstrating the importance of the conservation of these post-transcriptional gene regulation mechanisms (Lee et al., 1993; Chen et al., 2014). In fact, possible miRNAs-like molecules present in vesicles secreted by $P$. brasiliensis (Pb18) were already predicted using a strategy based on sequence similarity (Peres da Silva et al., 2015). Similarly, 22 miRNA-like sequences were identified in the genome of Humulus lupulus employing in silico prediction of miRNAs based on similar sequences present in the miRBase database (Mishra et al., 2015), demonstrating the efficiency of this strategy to characterize microRNAs.

Potential miRNAs-like molecules predicted in this work originated from precursors that form hairpin structures. During the formation of a mature miRNA, precursor molecules generate hairpins that are subsequently processed by dicer to form a miRNA/miRNA* duplex (Bartel et al., 2004). Previous reports demonstrated negative free folding energies of the precursors of PM-milR-M1 and PM-milR-M2 from P. marneffei of $-17.86 \mathrm{kcal} / \mathrm{mol}$ and $-23.88 \mathrm{kcal} / \mathrm{mol}$, respectively (Lau et al., 2013). In $A$. flavus, the values vary between $-19.4 \mathrm{kcal} / \mathrm{mol}$ and -140.2 $\mathrm{kcal} / \mathrm{mol}$, and in $M$. anisopliae they vary between -20 $\mathrm{kcal} / \mathrm{mol}$ and $-105.32 \mathrm{kcal} / \mathrm{mol}$ (Bai et al., 2015; Zhou et al., 2012).In this context, the values of negative free folding energy described in the present work are in agreement to those described in fungi. Interestingly, we obtained sequences of microRNA-like precursors of similar size to those described for other fungi (Zhou et al., 2012a,b). For example, in C. neoformans the sizes of the precursors of the $\mathrm{miR} 1$ and miR2 are approximately 70 nucleotides (Jiang et al., 2012).

Additionally we found low MFEI and similar AMFE values in the sequences of the microRNA-like precursors. Although these indices have not been described for miRNA-like precursors present in fungi, we hypothesize that the observed differences may be a result of the lower base complementarity between the precursor sequences present in fungal genomes when compared to plants, where the complementarity of bases could be higher (Mishra et al., 2015). In general, the data also demonstrate a higher amount of uracil in microRNA-like precursors, as already described in the literature (Zhang et al., 2006).

The experimental validation of some like microRNAs-like molecules in this work allowed the characterization of sequences ranging in size from 26 to $57 \mathrm{bp}$. In other fungi, smaller sizes for mature microRNAs-like were seen, as for example in $N$. crassa (Lee et al., 2010), $C$. neoformans (Jiang et al., 2012), and P. marneffei (Lau et al., 2013), in which detection of microRNAs-like molecules was performed by northern blot. In this work, the poly(T) adaptor RT-PCR method (Balcells et al., 2011) adds a polyA tail to the microRNA-like molecule, and possibly, this process confers a larger size to the mature microRNA-like.

In conclusion, our data demonstrate the conservation of proteins involved in the post-transcriptional mechanism of gene regulation in different species of the Paracoccidioides complex. The transcripts encoding these proteins were detected in the parasitic phase, and sequences of microRNAs-like molecules were seen to be conserved in $P$. brasiliensis. Furthermore, five micro-RNAs-like sequences were confirmed by qualitative RT-PCR. Hence, our results point to the ability of Paracoccidioides brasiliensis to produce microRNAs-like molecules. Several studies demonstrate the plasticity of fungi for surviving in different conditions. For example, during dimorphic transition, these pathogens alter the constitution of the cell wall and membrane, changing metabolic pathways for energy production (Felipe et al., 2005; Nunes et al., 2005; Bastos et al., 2007; Rezende et al., 2011). The molecular mechanisms that control these processes are still not fully elucidated, but the confirmation of the capacity of these fungi to produce microRNAs-like allow the investigation of function of these molecules in the regulation of biological processes essential for the survival of these pathogens under different conditions, such as those found in the host, or between the different morphological fungal stages.

\section{Acknowledgments}

This work was supported by grants from Conselho Nacional de Desenvolvimento Científico e Tecnológico (CNPq) and Fundação de Amparo à Pesquisa do Estado de Goiás (FAPEG), Instituto Nacional de Ciência e Tecnologia (INCT) de Estratégias de Interação PatógenoHospedeiro, and Newton Fund. JSC and MPB received fellowships from Coordenação de Aperfeiçoamento de Pessoal de Nível Superior Brazil (CAPES) - Finance Code 001.

\section{Conflict of interest}

The authors declare that they have no conflicts of interest related to the subject matter or materials discussed in this article.

\section{Author contributions}

CMA Soares and JS de Curcio conceived and designed the experiments. JS de Curcio, MP Batista performed the experiments. JS de Curcio, MP Batista, JD Paccez, E Novaes and CMA Soares analyzed and/or interpreted the data. CMA Soares contributed to reagents and materials. JS de Curcio, MP Batista, JD Paccez, E Novaes and CMA Soares wrote the manuscript.

\section{References}

Bai Y, Lan F, Yang W, Zhang F, Yang K, Li Z, Gao P and Wang S (2015) SRNA profiling in Aspergillus flavus reveals differ- 
entially expressed miRNA-like RNAs response to water activity and temperature. Fungal Genet Biol 81:113-119.

Balcells I, Cirera S and Busk PK (2011) Specific and sensitive quantitative RT-PCR of miRNAs with DNA primers. BMC Biotechnology 11:70.

Bartel DP, Lee R and Feinbaum R (2004) MicroRNAs: Genomics, biogenesis, mechanism, and function. Cell 116:281-97.

Bastos KP, Bailão AM, Borges CL, Faria FP, Felipe MSS, Silva MG, Martins WS, Fiúza RB, Pereira M and Soares CMA (2007) The transcriptome analysis of early morphogenesis in Paracoccidioides brasiliensis mycelium reveals novel and induced genes potentially associated to the dimorphic process. BMC Microbiology 7:29.

Bookout AL, Cummins CL, Mangelsdorf DJ, Pesola JM, Kramer MF, Pesola JM, Mangelsdorf DJ, Pesola JM and Kramer MF (2006) High-Throughput Real-Time Quantitative Reverse Transcription PCR. Current Protocols in Molecular Biology. John Wiley \& Sons, Hoboken, unit 15.8.

Camacho C, Coulouris G, Avagyan V, Ma N, Papadopoulos J, Bealer K and Madden TL (2009) BLAST+: Architecture and applications. BMC Bioinformatics 10:421.

Chen R, Jiang N, Jiang Q, Sun X, Wang Y, Zhang H and Hu Z (2014) Exploring microRNA-like small RNAs in the filamentous fungus Fusarium oxysporum. PLoS One 9:e104956.

Chiba M, Kimura M and Asari S (2012) Exosomes secreted from human colorectal cancer cell lines contain mRNAs, microRNAs and natural antisense RNAs, that can transfer into the human hepatoma HepG2 and lung cancer A549 cell lines. Oncol Rep 28:1551-1558.

Croston TL, Nayak AP, Lemons AR, Goldsmith WT, Gu JK, Germolec DR, Beezhold DH and Green BJ (2016) Influence of Aspergillus fumigatus conidia viability on murine pulmonary microRNA and mRNA expression following subchronic inhalation exposure. Clin Exp Allergy 46:1315-1327.

Croston TL, Lemons AR, Beezhold DH and Green BJ (2018) MicroRNA regulation of host immune responses following fungal exposure. Front Immunol 9:170

Dahlmann TA and Kück U (2015) Dicer-dependent biogenesis of small RNAs and evidence for microRNA-like RNAs in the penicillin producing fungus Penicillium chrysogenum. PLoS One 10:e0125989

Dehury B, Panda D, Sahu J, Sahu M, Sarma K, Barooah M, Sen P and Modi MK (2013) In silico identification and characterization of conserved miRNAs and their target genes in sweet potato (Ipomoea batatas L.) Expressed Sequence Tags (ESTs). Plant Signal Behav 8:1-13.

Dix A, Czakai K, Leonhardt I, Schäferhoff K, Bonin M, Guthke R, Einsele H, Kurzai O, Löffler J and Linde J (2017) Specific and novel microRNAs are regulated as response to fungal infection in human dendritic cells. Front Microbiol 8:270

Dlakic M (2006) DUF283 domain of Dicer proteins has a double-stranded RNA-binding fold. Bioinformatics 22:2711-2714.

Drinnenberg IA, Weinberg DE, Xie KT, Mower JP, Wolfe KH, Fink GR and Bartel DP (2009) RNAi in budding yeast. Science 326:544-550.

Felipe MSS, Andrade RV, Arraes FBM, Nicola AM, Maranhão AQ, Torres FAG, Silva-Pereira I, Poças-Fonseca MJ, Campos ÉG, Moraes LMP et al. (2005) Transcriptional profiles of the human pathogenic fungus Paracoccidioides brasiliensis in mycelium and yeast cells. J Biol Chem 280:24706-24714.

Finn RD, Coggill P, Eberhardt RY, Eddy SR, Mistry J, Mitchell AL, Potter SC, Punta M, Qureshi M, Sangrador-Vegas A et al. (2015) The Pfam protein families database: Towards a more sustainable future. Nucleic Acids Res 44:D279-D285.

Galagan JE, Calvo SE, Borkovich KA, Selker EU, Read ND, Jaffe D, FitzHugh W, Ma LJ, Smirnov S, Purcell S et al. (2003) The genome sequence of the filamentous fungus Neurospora crassa. Nature 422:859-868.

Grimson A, Srivastava M, Fahey B, Woodcroft BJ, Chiang HR, King N, Degnan BM, Rokhsar DS and Bartel DP (2008) Early origins and evolution of microRNAs and Piwiinteracting RNAs in animals. Nature 455:1193-1197.

He H, Jazdzewski K, Li W, Liyanarachchi S, Nagy R, Volinia S, Calin GA, Liu CG, Franssila K, Suster S et al. (2005) The role of microRNA genes in papillary thyroid carcinoma. Proc Natl Acad Sci U S A 102:19075-19080.

Janbon G, Maeng S, Yang DH, Ko Y, Jung KW, Moyrand F, Floyd A, Heitman J and Bahn YS (2010) Characterizing the role of RNA silencing components in Cryptococcus neoformans. Fungal Genet Biol 47:1070-80.

Jiang N, Yang Y, Janbon G, Pan J and Zhu X (2012) Identification and functional demonstration of miRNAs in the fungus Cryptococcus neoformans. PLoS One 7:e52734.

Joshua-Tor L (2006) The Argonautes. Cold Spring Harb Symp Quant Biol 71:67-72.

Kang K, Zhong J, Jiang L, Liu G, Gou CY, Wu Q, Wang Y, Luo J and Gou D (2013) Identification of microRNA-Like RNAs in the filamentous fungus Trichoderma reesei by Solexa sequencing. PLoS One 8:e76288.

Larkin MA, Blackshields G, Brown NP, Chenna R, Mcgettigan PA, McWilliam H, Valentin F, Wallace IM, Wilm A, Lopez $\mathrm{R}$ et al. (2007) Clustal $\mathrm{W}$ and Clustal $\mathrm{X}$ version 2.0. Bioinformatics 23:2947-2948.

Lau SKP, Chow WN, Wong AYP, Yeung JMY, Bao J, Zhang N, Lok S, Woo PCY and Yuen KY (2013) Identification of microRNA-like RNAs in mycelial and yeast phases of the thermal dimorphic fungus Penicillium marneffei. PLoS Negl Trop Dis 7:e2398.

Lee HCC, Li L, Gu W, Xue Z, Crosthwaite SK, Pertsemlidis A, Lewis ZA, Freitag M, Selker EU, Mello CC et al. (2010) Diverse pathways generate microRNA-like RNAs and Dicerindependent small interfering RNAs in fungi. Mol Cell 38:803-814.

Lee RC, Feinbaum RL and Ambros V (1993) The C. elegans heterochronic gene lin-4 encodes small RNAs with antisense complementarity to lin-14. Cell 75:843-854.

Lingel A, Simon B, Izaurralde E and Sattler M (2003) Structure and nucleic-acid binding of the Drosophila Argonaute 2 PAZ domain. Nature 426:465-469.

Lorenz R, Bernhart SH, Höner zu Siederdissen C, Tafer H, Flamm C, Stadler PF and Hofacker IL (2011) ViennaRNA Package 2.0. Algorithms Mol Biol 6:26.

Lorenz R, Wolfinger MT, Tanzer A and Hofacker IL (2016) Predicting RNA secondary structures from sequence and probing data. Methods 103:86-98.

Marioto DTG, Ferraro ACNS, Andrade FG, Oliveira MB, Itano EN, Petrofeza S and Venancio EJ (2017) Study of differential expression of miRNAs in lung tissue of mice submitted to experimental infection by Paracoccidioides brasiliensis. Med Mycol 55:774-784. 
Martinez R (2017) New trends in Paracoccidioidomycosis epidemiology. J Fungi 3:1.

Mishra AK, Duraisamy GS, Tycová A and Matouek J (2015) Computational exploration of microRNAs from expressed sequence tags of Humulus lupulus, target predictions and expression analysis. Comput Biol Chem 59:131-141.

Nakayashiki H (2005) RNA silencing in fungi: mechanisms and applications. FEBS Lett 579:5950-5957.

Nakayashiki H, Kadotani N and Mayama S (2006) Evolution and diversification of RNA silencing proteins in fungi. J Mol Evol 63:127-135.

Nunes LR, Oliveira RC, Batista D, Schmidt V, Marques R, Eliana M, Duarte DC, Ângelo L, Bernardes DS, Helena M et al. (2005) Transcriptome analysis of Paracoccidioides brasiliensis cells undergoing mycelium-to-yeast transition. Eukaryot Cell 4:2115-2128.

Peres da Silva R, Puccia R, Rodrigues ML, Oliveira DL, Joffe LS, César GV, Nimrichter L, Goldenberg S and Alves LR (2015) Extracellular vesicle-mediated export of fungal RNA. Sci Rep 5:7763.

Quinlan AR and Hall IM (2010) BEDTools: A flexible suite of utilities for comparing genomic features. Bioinformatics 26:841-842.

Restrepo A (1985) The ecology of Paracoccidioides brasiliensis: A puzzle still unsolved. Sabouraudia 23:323-34.

Rezende TCV, Borges CL, Magalhães AD, Sousa MV, Ricart CAO, Bailão AM and Soares CMA (2011) A quantitative view of the morphological phases of Paracoccidioides brasiliensis using proteomics. J Proteomics 75:572-587.

Saitou N and Nei M (1987) The neighbor-joining method: A new method for reconstructing phylogenetic trees. Mol Biol Evol 4:406-25.

Sharma S, Kar S, Sahu SK, Samal B, Mallick A and Das S (2010) Is inclusion of Sabouraud dextrose agar essential for the laboratory diagnosis of fungal keratitis? Indian J Ophthalmol 58:281.

Silva-Bailão MG, Bailão EFLC, Lechner BE, Gauthier GM, Lindner H, Bailão AM, Haas H and Soares CMDA (2014) Hydroxamate production as a high affinity iron acquisition mechanism in Paracoccidioides spp. PLoS One 9:e105805.

Song JJ, Smith SK, Hannon GJ and Joshua-Tor L (2004) Crystal structure of Argonaute and its implications for RISC slicer activity. Science 305:1434-1437.

Thompson JD, Gibson TJ, Plewniak F, Jeanmougin F and Higgins DG (1997) The CLUSTAL X windows interface: Flexible strategies for multiple sequence alignment aided by quality analysis tools. Nucleic Acids Res 25:4876-4882.

Tsutsumi A, Kawamata T, Izumi N, Seitz H and Tomari Y (2011) Recognition of the pre-miRNA structure by Drosophila Dicer-1. Nat Struct Mol Biol 18:1153-1158.

Turissini DA, Gomez OM, Teixeira MM, McEwen JG and Matute DR (2017) Species boundaries in the human pathogen Paracoccidioides. Fungal Genet Biol 106:9-25.
Yan KS, Yan S, Farooq A, Han A, Zeng L and Zhou MM (2003) Structure and conserved RNA binding of the PAZ domain. Nature 426:468-74.

Zhang BH, Pan XP, Cox SB, Cobb GP and Anderson TA (2006) Evidence that miRNAs are different from other RNAs. Cell Mol Life Sci 63:246-254.

Zhang H, Kolb FA, Jaskiewicz L, Westhof E and Filipowicz W (2004) Single processing center models for human Dicer and bacterial RNase III. Cell 118:57-68.

Zhao T, Li G, Mi S, Li S, Hannon GJ, Wang XJ and Qi Y (2007) A complex system of small RNAs in the unicellular green alga. Genes Dev 1190-1203.

Zhou J, Fu Y, Xie J, Li B, Jiang D, Li G and Cheng J (2012a) Identification of microRNA-like RNAs in a plant pathogenic fungus Sclerotinia sclerotiorum by high-throughput sequencing. Mol Genet Genomics 287:275-282.

Zhou Q, Wang Z, Zhang J, Meng H and Huang B (2012b) Genome-wide identification and profiling of microRNA-like RNAs from Metarhizium anisopliae during development. Fungal Biol 116:1156-62.

\section{Internet Resources}

Uniprot database,(http://www.uniprot.org/)

NCBI database, (http://blast.ncbi.nlm.nih.gov/Blast.cgi?PAGE=Proteins) PFAM database, (http://pfam.xfam.org/).

\section{Supplementary material}

The following online material is available for this article:

Figure S1 - Flowchart of study design.

Figure S2 - Phylogenetic tree of the dicer and argonaute proteins.

Figure S3 - Aligment analysis of miRNAs-like in the genome of $P$. brasilensis.

Table S1 - Access numbers of the proteins used to construct the phylogenetic trees

Table S2 - Oligonucleotide sequences used in the present study.

Table S3 - miRNAs-like homologs to other fungi species identified in the genome of $P$. brasiliensis Pb18.

Table S4 - Identification of miRNAs-like described in fungi vesicles with identity in the genome of $P$. brasiliensis $\mathrm{Pb} 18$.

Associate Editor: Juan Lucas Argueso Almeida

License information: This is an open-access article distributed under the terms of the Creative Commons Attribution License (type CC-BY), which permits unrestricted use, distribution and reproduction in any medium, provided the original article is properly cited. 